\title{
Gecko Active Components Regulates Endoplasmic Reticulum Stress to Induce the Apoptosis of KYSE150 Cells through PERK Pathway
}

\author{
Yimeng Duan, Lengxin Duan, Zeyue Huang, \\ Bingbing Wang, Jiangang Wang* \\ Department of Pharmacology, Medical College, Henan University of Science and Technology, \\ Luoyang, China \\ Email:*ylwjg@163.com
}

How to cite this paper: Duan, Y.M., Duan, L.X., Huang, Z.Y., Wang, B.B. and Wang, J.G. (2018) Gecko Active Components Regulates Endoplasmic Reticulum Stress to Induce the Apoptosis of KYSE150 Cells through PERK Pathway. Journal of Biosciences and Medicines, 6, 36-44. https://doi.org/10.4236/jbm.2018.65005

Received: February 10, 2018

Accepted: May 18, 2018

Published: May 21, 2018

\begin{abstract}
Objective: Gecko active components (GACs), extracted from the powder of whole Gecko, have been reported to be effective against esophageal squamous cell carcinoma (ESCC). Endoplasmic reticulum stress (ERs) has been regarded as an important cause for pathogenesis of esophageal squamous cell carcinoma (ESCC). In this paper, we aimed to study the effect of GACS on apoptosis of human esophageal carcinoma KYSE150 cells and to analyze the underlying signaling pathway. Methods: MTT assay was used to detect the viability of KYSE150 cells, and Flow cytometry was applied to detect reactive oxygen species (ROS), calcium generation, and the level of mitochondrial membrane potential (MMP). Western blot analysis was applied to observe the expression of apoptosis-related proteins and endoplasmic reticulum stress (ERs)-related proteins in KYSE150 cells. Results: The results showed that GACs inhibited KYSE150 cell vitality in a dose- and time-dependent manner. Not only that, GACs could up-regulated ERs-related and apoptosis-related proteins expression, and the content of ROS and calcium were significantly increased, and also, the level of MMP was significantly decreased. Conclusion: The results of this report suggested that induction of apoptosis occurs through the ERs dependent signaling pathways.
\end{abstract}

\section{Keywords}

Gecko Active Components, Esophageal Squamous Cell Carcinoma, Endoplasmic Reticulum Stress, Anti-Tumor 


\section{Introduction}

Esophageal cancer is the eighth most common cancer and the sixth most common causes of cancer mortality worldwide, and the incidence of ESCC in China is much higher than other countries. Esophageal cancer comprises two histological types: esophageal squamous cell carcinoma (ESCC) and esophageal adenocarcinoma (EAD) [1] [2]. The five-year survival rate of ESCC is below $20 \%$, and the exact molecular mechanism and pathological process of ESCC remain poorly elucidated although numerous efforts had been made. Figuring out the molecular mechanisms of carcinogenesis, metastasis and invasion in ESCC will help improving efficiency of ESCC therapy [3].

Gecko has been used as anti-tumor medicine for many years. Gecko active components (GACs) are extracted from gecko that exhibits a variety of pharmacological activities. Gecko active components (GACs) have been shown to be highly curative effect in some cell and animal tests. It has been previously revealed that GACs displays potent antitumor activities in various types of cancer, including liver cancer, cervical cancer, and laryngeal cancer [4] [5], but the effects of GACs on human ESCC and its mechanism of action have not been elucidated.

In eukaryotic cells, the endoplasmic reticulum(ER) is essential for the regulation of calcium homeostasis, protein synthesis, and proper protein folding. A disturbance of $\mathrm{ER} \mathrm{Ca}^{2+}$ homeostasis or the protein process can contribute to ER stress, which in turn induces the production of ROS in the ER and mitochondria. High ROS generation induces the opening of the mitochondrial permeability transition pore (mPTP) [6] [7] [8]. To cope with these hostile environments, unfolded protein response(UPR) is activated to restore ER proteostasis via three endoplasmic reticulum membrane sensor protein, including the protein kinase RNA-like ER kinase (PERK), inositol-requiring kinase 1 (IRE1), and activating transcription factor 6 (ATF6). Since, a number of proteins that regulate apoptosis become involved, and leading to cell death [9] [10]. However, the effects of signaling in the anti-ESCC actions of GACs have not been examined. In the present study, we assessed the anticancer activity of GACs in ESCC and explored the role of ERS signaling in GACs treatment.

\section{Material and Methods}

\subsection{Preparation of Gecko Active Components (GACs)}

The whole-dried Gecko was purchased from Anhui Bozhou Yong gang Co. Ltd (Bozhou, China). Gecko powders (100 g) were mixed with $400 \mathrm{ml}$ double-distilled water and made into homogenate. Following centrifugation at $5600 \times \mathrm{g}$ for 5 $\mathrm{min}$, the precipitation was collected and soaked in $400 \mathrm{ml}$ of $55 \%$ ethanol solution. The supernatant was obtained following centrifugation at $5600 \times \mathrm{g}$ for 5 min, and was evaporated under reduced pressure at $55^{\circ} \mathrm{C}$. Subsequently, yellow powders were collected following freeze-drying of the residue liquid. Gel filtration chromatography (Sephadex G-25) was used to purify the yellow powders, 
and ultimately, the GACs were collected.

\subsection{CELL Line and Cultures}

The human esophageal squamous cell carcinoma KYSE150 cells were kindly provided by the First Affiliated Hospital of Henan University of Science and Technology. KYSE150 cell was grown in RPMI-1640 medium (Solarbio, Beijing, China) with $10 \%$ fetal bovine serum (FBS) at $37^{\circ} \mathrm{C}$ in a humidified $5 \% \mathrm{CO}_{2}$ incubator. The culture medium was replaced every 2 days and the cells in the exponential growth phase were collected for the following experiments.

\subsection{MTT Assay}

KYSE150 cells were seeded in a 96-well plate at a density of $2.5 \times 104$ cells/well. The cells were exposed to different doses of GACs $(0.1,0.15,0.2,0.25,0.3,0.35$, $\left.0.4,0.45,0.5 \mathrm{mg} \cdot \mathrm{mL}^{-1}\right)$. After incubation for 20,44 , and $68 \mathrm{~h}$, respectively, $20 \mu \mathrm{l}$ of the MTT reagent $(5 \mathrm{mg} / \mathrm{l})$ was added per well and incubated for another $4 \mathrm{~h}$. The supernatant was replaced by $200 \mu$ dimethyl sulfoxide (DMSO) and the absorbance (A) was measured with an ELX800 Universal Microplate reader at 490 $\mathrm{nm}$. The cell proliferation inhibition rate (IR) was as follows: IR = (1-A GPM/A control) $\times 100 \%$.

\subsection{Determination of Intracellular Reactive Oxygen Species (ROS), Calcium, and Mitochondrial Membrane Potential (MMP)}

The intracellular ROS levels were measured using a Reactive Oxygen Species Assay Kit (Solarbio, Beijing, China); the level of intracellular calcium was performed using calcium fluorescence probe kit (Beyotime Biotechnology, Shanghai, China); MMP changes were confirmed by the fluorescent probe JC-1 (Solarbio, Beijing, China).

KYSE150 cells $\left(2 \times 10^{5}\right)$ were plated in 6-well plates and cultured overnight. The cells were treated with different concentrations of GACs $(0.1,0.15,0.225$ $\left.\mathrm{mg} \cdot \mathrm{mL}^{-1}\right)$. After treatment, the cells were harvested and then washed twice with cold-phosphate buffered saline (PBS). Next, the cells were incubated with 10 $\mu \mathrm{mol} \cdot \mathrm{L}^{-1}$ of DCF-DA for $20 \mathrm{~min}, 10 \mu \mathrm{mol} \cdot \mathrm{L}^{-1}$ of calcium fluorescence probe for $40 \mathrm{~min}$, and JC-1 for $30 \mathrm{~min}$ at $37^{\circ} \mathrm{C}$ in darkness, respectively, followed by three washes with PBS. Finally, the samples were tested with a flow cytometry (Beckman).

\subsection{Western Blotting}

KYSE150 cells $\left(2.5 \times 10^{5}\right)$ in 6-well plates were treated with different concentration GACs $\left(0.1,0.15,0.225 \mathrm{mg} \cdot \mathrm{mL}^{-1}\right)$ for $24 \mathrm{~h}$. The cells were washed with ice-cold PBS, and $60 \mu \mathrm{l}$ RIPA lysate (Solarbio, Beijing, China) were added to all samples. The protein concentrations were detected by BCA protein assay kit (Solarbio, Beijing, China) and the proteins were separated by $12 \%$ SDS-PAGE and transferred to PVDF (poly-vinylidene difluoride transfer, Millipore, USA) membranes. The membranes were blocked with $5 \%$ skimmed milk (BD, USA) 
for $2 \mathrm{~h}$ at $37^{\circ} \mathrm{C}$, and then incubated with primary antibodies, anti-PERK, anti-GRP78, anti-ATF4, anti-CHOP, anti-PARP(poly ADP-ribose polymerase), anti-caspase-3(cysteinyl aspartate specific proteinase-3) and anti-GAPDH overnight at $4^{\circ} \mathrm{C}$. On the following day, HRP-labeled secondary antibodies (goat anti-rabbit) were added for incubation in $37^{\circ} \mathrm{C}$ for $1 \mathrm{~h}$. All the antibodies were purchased from Proteintech (Wuhan, China). The eECL western blot kit (CWBIOTECH, Beijing, China) was used to detect the signals (BioRad, USA). The results of the western blots were analyzed using Image J software.

\subsection{Statistical Analysis}

The experimental results presented in the figures are representative of three or more independent observations. Values were showed as mean \pm SD (standard deviation). One-way ANOVA was applied to analyze the data by SPSS 19.0 system to determine differences between groups. Values of $\mathrm{P}<0.05$ were considered to be statistically significant.

\section{Results}

\subsection{Effects of GACs on the Proliferation and Migration Ability of KYSE150 Cells}

KYSE150 cells were exposed to different concentrations of GACs to evaluate its survival rates using MTT method. Time-and dose-effect curve of GACs on KYSE150 cells were shown in Figure 1, it can be seen that GACs inhibit the cell vitality of KYSE150 cells with the increase of concentration and the extension of time. The half-maximal inhibitory concentration $\left(\mathrm{IC}_{50}\right)$ values of GACs at $24 \mathrm{~h}$, $48 \mathrm{~h}, 72 \mathrm{~h}$ were $0.2223,0.1849,0.1493 \mathrm{mg} \cdot \mathrm{mL}^{-1}$, respectively.

\subsection{Effects of GACs Treatment on ROS, Calcium and MMP Levels in Human ESCCs}

Excessive ROS production has been shown to induce apoptosis in various types of cancer cells. As shown in Figure 2(a), treatment with GACs caused dose-dependent increases in ROS production in ESCC cells. The mean fluorescence intensity of different concentrations GACs on HepG2 cells were (74.95 \pm $10.56)$, (173.22 \pm 15.23$),(442.43 \pm 10.23)$, and the mean fluorescence intensity of the control group was (44.44 \pm 8.26$)$. Compared with control group, the content of ROS was significantly increased. These results suggested that ROS accumulation may be responsible for inducing ESCC cells apoptosis. Calcium communicates with a number of other systems and pathways, among them also with reactive oxygen species (ROS). As shown in Figure 3B, treatment with GACs caused dose-dependent increases in calcium production in ESCC cells. The mean fluorescence intensity of different concentrations GACs on HepG2 cells were (35.93 $\pm 5.36),(55.28 \pm 8.24),(135.81 \pm 10.56)$, and the mean fluorescence intensity of the control group was $(17.12 \pm 2.32)$. Compared with control group, the level of calcium was significantly increased. Figure $2(\mathrm{c})$ is statistical analysis of data from (a) and (b). Previous studies showed that ROS generation was closely 


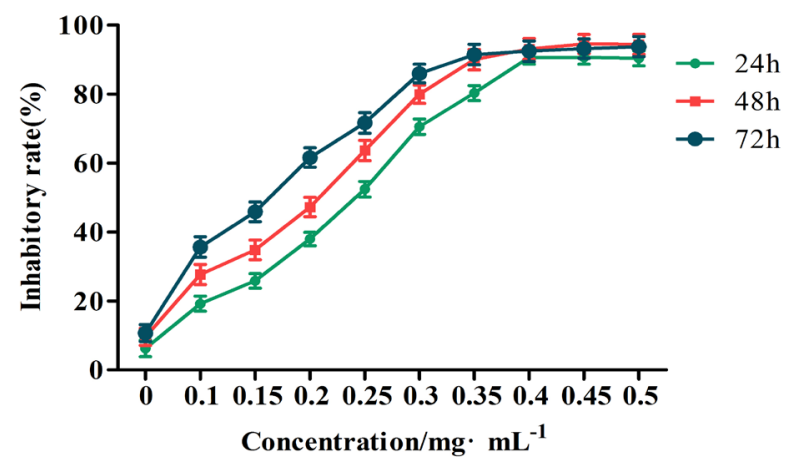

Figure 1. The anti-proliferative effect of GACs on KYSE150 cells. The growth inhibitory effect of GACs on KYSE150 cells were assessed by MTT assay in vitro.
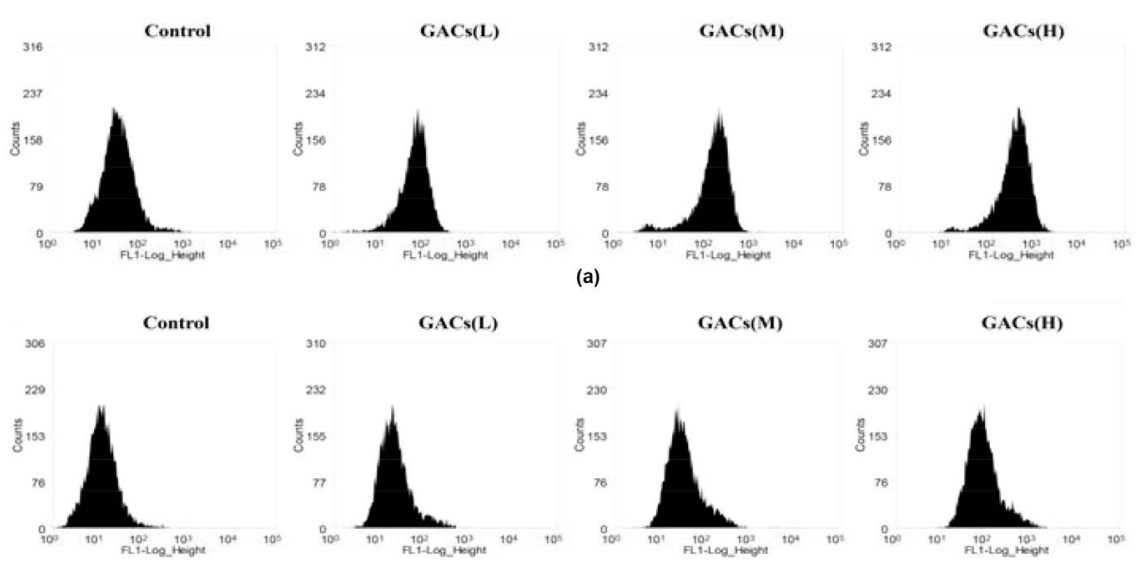

(b)

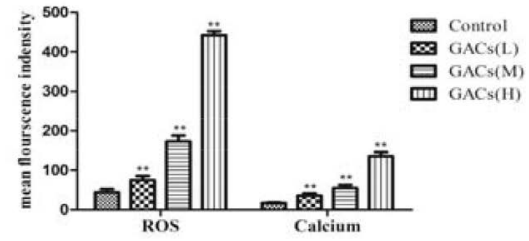

(c)

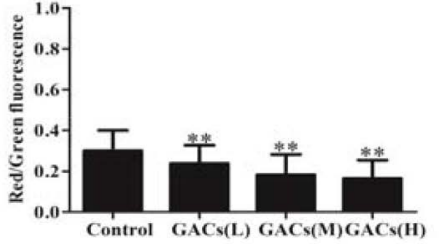

(d)

Figure 2. Effect of GACs on ROS generation, calcium levels and MMP dissipation in KYSE150 cells. (a) KYSE150 cells were treated with indicated concentration of GACs for $24 \mathrm{~h}$. KYSE150 cells were incubated with GACs in a dose- and time-dependent manner as indicated and ROS generation was determined by staining the cells with DCFH-DA; (b) KYSE150 cells were treated with GACs for $24 \mathrm{~h}$ and intracellular calcium levels was measured according to kit instructions; (c) Statistical analysis of data from a and b; (d) GACs caused a disruption of MMP in KYSE150 cells, as evidenced by an increased proportion of cells with green fluorescent light and a decrease proportion of cells with a higher red (JC-1 aggregates)/green (JC-1 monomers) ratio of JC-1 fluorescence. Abbreviations as above. Values are mean $\pm \mathrm{SD}(\mathrm{n}=5)$. Compared with control group, ${ }^{\star} P<0.05$, ${ }^{* *} P<0.01$.

related to MMP changes. As shown in Figure 2(d), the number of depolarized cells was increased massively after exposure to various concentrations of GACs, which indicated that mitochondrial function was dose-dependently disturbed by 
GACs treatment. The MMP of different concentrations GACs on HepG2 cells were $(0.24 \pm 0.09),(0.18 \pm 0.07),(0.16 \pm 0.08)$, the MMP of control group was $(0.31 \pm 0.11)$. Compared with the control group, the level of MTP was significantly decreased.

\subsection{Effects of GACs on ERs Signaling and Apoptosis Related Proteins in Human ESCC Cells}

The results of ROS may illustrate the activation of ERs. Western blots revealed that GACs treatment up-regulated PERK, GRP78, ATF4 and CHOP expression levels in KYSE150 cells in a dose-dependent manner (Figure 3(a)). Figure 3(b) is statistical analysis of data from (a). Additionally, we determined whether GACs affects the apoptotic signaling pathway in KYSE150 cells by measuring the protein expression of PARP (poly ADP-ribose polymerase), Caspase3 (cysteinyl aspartate specific proteinase-3). GACs treatment increased the expression of PARP, Caspase3 (Figure 3(c)). Figure 3(d) is statistical analysis of data from (c). All data revealed that the anti-ESCC effect of GACs was mediate by ERs.

\section{Discussion}

ESCC is characterized by a poor prognosis. The accompanying drug resistance and side effects substantially weaken the therapeutic effectiveness [11]. Recently reports have revealed that some traditional Chinese products and their extract can be used to develop effective anticancer drugs. Some previous studies showed that gecko extract exerted strong anti-tumor effects by inducing apoptosis; however, the effects of GACs on ESCC cells have never been investigated, and the underlying mechanism of the anticancer effect is still unclear. In the present study, we demonstrated that GACs could significantly inhibit the proliferation of KYSE150 cells in a dose-and time-dependent manners via MTT assay, and we found that GACs induces apoptosis in ESCC cells via ROS generation, calcium production, and mitochondrial dysfunction, meanwhile ERs is activated by ROS generation.

Previous studies have found that cancer cells contain higher levels of ROS than those of normal cells as a result of their hypermetabolism, which is closely associated with cell proliferation, differentiation and cell death [12] [13] [14]. Our results showed that GACs increased ROS levels of KYSE150 cells in a time-dependent manner. Calcium is an important second messenger involved in intra- and extracellular signaling cascades and plays an essential role in cell life and death decisions. ROS can significantly affect calcium influx into the cell and intracellular calcium stores. Our results showed that GACs increased calcium levels of KYSE150 cells in a time-dependent manner. Additionally, ROS could more directly damage MMP by provoking mitochondrial membrane hyperpolarisation [15]. A number of reports has been demonstrated that apoptosis is related to MMP depolarization and that the permeabilization of outer mitochondrial membranes is a critical step in apoptosis [16] [17]. Our results demonstrated that GACs induced MMP depolarization. 


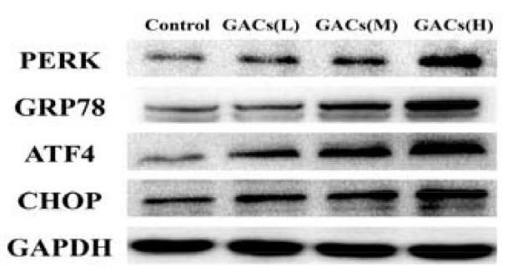

(a)

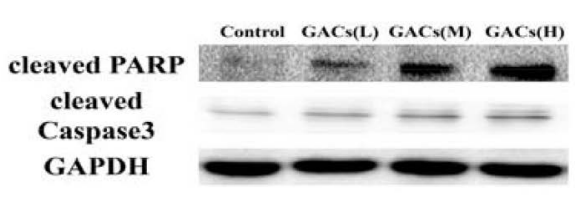

(c)

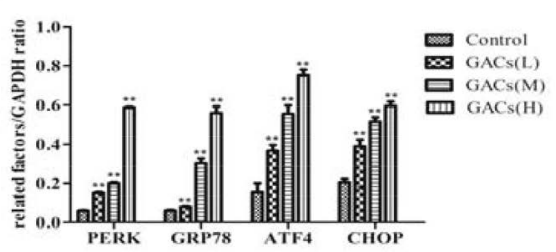

(b)

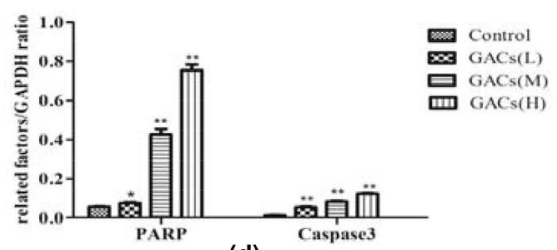

(d)

Figure 3. Effect of GACs on ERs signaling and apoptosis related proteins in human ESCC cells. (a) KYSE150 cells were treated with GACs for $24 \mathrm{~h}$ and expression of PERK, GRP78, ATF4 and CHOP was measured by Western blot; (b) Statistical analysis of data from (b); (c) KYSE150 cells were treated with indicated concentrations of GACs for $24 \mathrm{~h}$ and expressions of cleaved PARP and cleaved caspases- 3 were measured by Western blot analysis; (d) Statistical analysis of data from (c). Changes were significantly different compared with control group $\left({ }^{\star} P<0.05,{ }^{* *} P<0.01\right)$. GAPDH was used as the internal loading control.

The ER is highly mediated protein folding, and only correctly folded proteins performed their physiological functions. Due to their high growth and proliferation rates, cancer cells require an increased rate of protein folding and assembly in the ER [18] [19]. In addition, some cancer cells express mutant proteins that cannot be correctly folded, which activates the UPR. The activation of unfolded protein response (UPR) plays a protective role in cells under ER stress. Stress condition such as ROS generation and calcium production can affect the ER steady state, trigger ERs and initiate the UPR [20]. Under ERs, the PERK pathway of cells was up-regulated, and then through phosphorylation of the translation initiation factor eIf2a to ameliorates ERs. The downstream protein ATF4 induced expression of the transcription of C/EBP homologous protein (CHOP), finally enabled the recovery of protein translation [21]. Apoptosis plays an essential role in maintaining human stable internal environment. ERs was involved in regulating cell apoptosis [22]. Recent studies have shown that ROS overexpression and MMP disruption are two early steps involved in mitochondria-mediated apoptosis [23]. Herein, our results suggested that the activation of ERs signaling is associated with the induction of the mitochondrial apoptotic pathway in human cancer cells.

In summary, the present findings demonstrate that GACs effectively induced apoptosis via ER stress pathway. Most importantly, our findings provide a foundation for GACs to become a potential anti-tumor agent in ESCC treatment.

\section{Acknowledgements}

This study was supported by Science and Technology Key Research Fund funded 
projects of Henan Province, China, No. 142102310031. We thank Dr Jian-gang Wang for their proofreading the article.

\section{References}

[1] Kang, X., Chen, K., Li, Y., Li, J., D’Amico, T.A. and Chen, X. (2015) Personalized Targeted Therapy for Esophageal Squamous Cell Carcinoma. World J Gastroenterol, 21, 7648-7658. https://doi.org/10.3748/wjg.v21.i25.7648

[2] Li, J.S., Ying, J.M., Wang, X.W., Wang, Z.H., Tao, Q. and Li, L.L. (2013) Promoter Methylation of Tumor Suppressor Genes in Esophageal Squamous Cell Carcinoma. Chin J Cancer, 32, 3-11. https://doi.org/10.5732/cjc.011.10381

[3] Song, Y., Li, L., Ou, Y., et al. (2014) Identification of Genomic Alterations in Oesophageal Squamous Cell Cancer. Nature, 509, 91-95.

https://doi.org/10.1038/nature13176

[4] Liu, F., Wang, J.G., Wang, S.Y., Li, Y., Wu, Y.P. and Xi, S.M. (2008) Antitumor Effect and Mechanism of Gecko on Human Esophageal Carcinoma Cell Lines In Vitro and Xenografted Sarcoma 180 in Kunming Mice. World J Gastroenterol, 14, 3990-3996. https://doi.org/10.3748/wjg.14.3990

[5] Jin, Y., Duan, L.X., Wang, J.G., et al. (2016) Mechanism of Apoptosis Induction in Human Hepatocellular Carcinoma Cells Following Treatment with a Gecko Peptides Mixture. Biomed Rep, 5, 73-78. https://doi.org/10.3892/br.2016.664

[6] Zorova, L.D., Popkov, V.A., Plotnikov, E.Y., et al. (2017) Mitochondrial Membrane Potential. Anal Biochem. https://doi.org/10.1016/j.ab.2017.07.009

[7] Fan, C., Yang, Y., Liu, Y., et al. (2016) Icariin Displays Anticancer Activity against Human Esophageal Cancer Cells via Regulating Endoplasmic Reticulum Stress-Mediated Apoptotic Signaling. Sci Rep, 6, 21145. https://doi.org/10.1038/srep21145

[8] Mazars, C., Thuleau, P., Lamotte, O. and Bourque, S. (2010) Cross-Talk between ROS and Calcium in Regulation of Nuclear Activities. Mol Plant, 3, 706-718. https://doi.org/10.1093/mp/ssq024

[9] Avril, T., Vauleon, E. and Chevet, E. (2017) Endoplasmic Reticulum Stress Signaling and Chemotherapy Resistance in Solid Cancers. Oncogenesis, 6, e373. https://doi.org/10.1038/oncsis.2017.72

[10] Wang, C.L., Liu, C., Niu, L.L., Wang, L.R., Hou, L.H. and Cao, X.H. (2013) Surfactin-Induced Apoptosis through ROS-ERS-Ca ${ }^{2+}$-ERK Pathways in HepG2 Cells. Cell Biochem Biophys, 67, 1433-1439. https://doi.org/10.1007/s12013-013-9676-7

[11] Liu, R.M., Sun, D.N., Jiao, Y.L., et al. (2017) Macrophage Migration Inhibitory Factor Promotes Tumor Aggressiveness of Esophageal Squamous Cell Carcinoma via Activation of Akt and Inactivation of GSK3beta. Cancer Lett.

[12] Liao, H.Y., Kao, C.M., Yao, C.L., Chiu, P.W., Yao, C.C. and Chen, S.C. (2017) 2,4,6-Trinitrotoluene Induces Apoptosis via ROS-Regulated Mitochondrial Dysfunction and Endoplasmic Reticulum Stress in HepG2 and Hep3B Cells. Sci Rep, 7, 8148. https://doi.org/10.1038/s41598-017-08308-Z

[13] Park, S., Park, J.A., Yoo, H., Park, H.B. and Lee, Y. (2017) Proteasome Inhibitor-Induced Cleavage of HSP90 Is Mediated by ROS Generation and Caspase 10-Activation in Human Leukemic Cells. Redox Biol, 13, 470-476. https://doi.org/10.1016/j.redox.2017.07.010

[14] Xian, M., Cao, H., Cao, J., Shao, X., et al. (2017) Bortezomib Sensitizes Human Osteosarcoma Cells to Adriamycin-Induced Apoptosis through ROS-Dependent Acti- 
vation of p-eIF2alpha/ATF4/CHOP Axis. Int J Cancer, 141, 1029-1041. https://doi.org/10.1002/ijc.30792

[15] Yeste, M., Estrada, E., Rocha, L.G., Marin, H., Rodriguez-Gil, J.E. and Miro, J. (2015) Cryotolerance of Stallion Spermatozoa Is Related to ROS Production and Mitochondrial Membrane Potential Rather than to the Integrity of Sperm Nucleus. Andrology, 3, 395-407. https://doi.org/10.1111/andr.291

[16] Sharaf, M.S., Stevens, D. and Kamunde, C. (2017) Zinc and Calcium Alter the Relationship between Mitochondrial Respiration, ROS and Membrane Potential in Rainbow Trout (Oncorhynchus mykiss) Liver Mitochondria. Aquat Toxicol, 189, 170-183. https://doi.org/10.1016/j.aquatox.2017.06.005

[17] Lee, D.G., Choi, B.K., Kim, Y.H., Oh, H.S., Park, S.H., Bae, Y.S. and Kwon, B.S. (2016) The Repopulating Cancer Cells in Melanoma Are Characterized by Increased Mitochondrial Membrane Potential. Cancer Lett, 382, 186-194.

https://doi.org/10.1016/j.canlet.2016.08.027

[18] Choi, A.Y., Choi, J.H., Hwang, K.Y., et al. (2014) Licochalcone A Induces Apoptosis Through Endoplasmic Reticulum Stress via a Phospholipase Cgamma1-, Ca(2+)-, and Reactive Oxygen Species-Dependent Pathway in HepG2 Human Hepatocellular Carcinoma Cells. Apoptosis, 19, 682-697.

https://doi.org/10.1007/s10495-013-0955-y

[19] Zhang, X., Yu, L. and Xu, H. (2016) Lysosome Calcium in ROS Regulation of Autophagy. Autophagy, 12, 1954-1955. https://doi.org/10.1080/15548627.2016.1212787

[20] Hotamisligil, G.S. (2010) Endoplasmic Reticulum Stress and the Inflammatory Basis of Metabolic Disease. Cell, 140, 900-917. https://doi.org/10.1016/j.cell.2010.02.034

[21] Li, S., Dong, P., Wang, J., Zhang, J., et al. (2010) Icariin, a Natural Flavonol Glycoside, Induces Apoptosis in Human Hepatoma SMMC-7721 Cells via a ROS/JNK-Dependent Mitochondrial Pathway. Cancer Lett, 298, 222-230. https://doi.org/10.1016/j.canlet.2010.07.009

[22] Yang, L., Wang, P., Wang, H., et al. (2013) Fucoidan Derived from Undaria pinnatifida Induces Apoptosis in Human Hepatocellular Carcinoma SMMC-7721 Cells via the ROS-Mediated Mitochondrial Pathway. Mar Drugs, 11, 1961-1976. https://doi.org/10.3390/md11061961

[23] Zhang, X., Tang, X., Wang, M., Zhang, W., Zhou, B. and Wang, Y. (2017) ROS and Calcium Signaling Mediated Pathways Involved in Stress Responses of the Marine Microalgae Dunaliella salina to Enhanced UV-B Radiation. J Photochem Photobiol $B$, 173, 360-367. https://doi.org/10.1016/j.jphotobiol.2017.05.038 